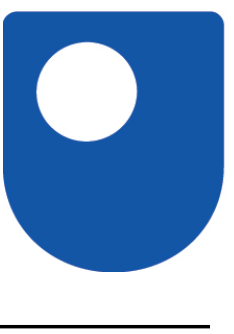

The Open University's repository of research publications and other research outputs

\title{
High precision astrometry mission for the detection and characterization of nearby habitable planetary systems with the Nearby Earth Astrometric Telescope (NEAT)
}

\section{Journal Item}

How to cite:

Malbet, Fabien; Léger, Alain; Shao, Michael; Goullioud, Renaud; Lagage, Pierre-Olivier; Brown, Anthony G. A.; Cara, Christophe; Durand, Gilles; Eiroa, Carlos; Feautrier, Philippe; Jakobsson, Björn; Hinglais, Emmanuel; Kaltenegger, Lisa; Labadie, Lucas; Lagrange, Anne-Marie; Laskar, Jacques; Liseau, René; Lunine, Jonathan; Maldonado, Jesús; Mercier, Manuel; Mordasini, Christoph; Queloz, Didier; Quirrenbach, Andreas; Sozzetti, Alessandro; Traub, Wesley; Absil, Olivier; Alibert, Yann; Andrei, Alexandre Humberto; Arenou, Frédéric; Beichman, Charles; Chelli, Alain; Cockell, Charles S.; Duvert, Gilles; Forveille, Thierry; Garcia, Paulo J. V.; Hobbs, David; Krone-Martins, Alberto; Lammer, Helmut; Meunier, Nadège; Minardi, Stefano; Moitinho de Almeida, André; Rambaux, Nicolas; Raymond, Sean; Röttgering, Huub J. A.; Sahlmann, Johannes; Schuller, Peter A.; Ségransan, Damien; Selsis, Franck; Surdej, Jean; Villaver, Eva; White, Glenn J. and Zinnecker, Hans (2012). High precision astrometry mission for the detection and characterization of nearby habitable planetary systems with the Nearby Earth Astrometric Telescope (NEAT). Experimental Astronomy, 34(2) pp. 385-413.

For guidance on citations see FAQs.

(C) 2011 Springer Science+Business Media B.V.

Version: Accepted Manuscript

Link(s) to article on publisher's website:

http://dx.doi.org/doi:10.1007/s10686-011-9246-1

Copyright and Moral Rights for the articles on this site are retained by the individual authors and/or other copyright owners. For more information on Open Research Online's data policy on reuse of materials please consult the policies page. 
oro.open.ac.uk 


\title{
High precision astrometry mission for the detection and characterization of nearby habitable planetary systems with the Nearby Earth Astrometric Telescope (NEAT)
}

\author{
Fabien Malbet • Alain Léger • Michael Shao • Renaud Goullioud • \\ Pierre-Olivier Lagage - Anthony G. A. Brown - Christophe Cara . \\ Gilles Durand · Carlos Eiroa • Philippe Feautrier • Björn Jakobsson · \\ Emmanuel Hinglais · Lisa Kaltenegger · Lucas Labadie • Anne-Marie \\ Lagrange • Jacques Laskar • René Liseau • Jonathan Lunine • Jesús \\ Maldonado - Manuel Mercier - Christoph Mordasini • Didier Queloz • \\ Andreas Quirrenbach • Alessandro Sozzetti - Wesley Traub • Olivier \\ Absil • Yann Alibert • Alexandre Humberto Andrei • Frédéric Arenou . \\ Charles Beichman • Alain Chelli • Charles S. Cockell • Gilles Duvert • \\ Thierry Forveille - Paulo J.V. Garcia • David Hobbs • Alberto \\ Krone-Martins • Helmut Lammer • Nadège Meunier • Stefano Minardi • \\ André Moitinho de Almeida • Nicolas Rambaux • Sean Raymond . \\ Huub J. A. Röttgering • Johannes Sahlmann • Peter A. Schuller • \\ Damien Ségransan • Franck Selsis • Jean Surdej • Eva Villaver • Glenn \\ J. White • Hans Zinnecker \\ Received: date / Accepted: date
}

\begin{abstract}
A complete census of planetary systems around field of view located $40 \mathrm{~m}$ away from the telescope and a volume-limited sample of solar-type stars (FGK dwarfs) in the Solar neighborhood $(d \leq 15 p c)$ with uniform sensitivity down to Earth-mass planets within their Habitable Zones out to several AUs would be a major milestone in extrasolar planets astrophysics. This fundamental goal can be achieved with a mission concept such as NEAT — the Nearby Earth Astrometric Telescope.

NEAT is designed to carry out space-borne extremelyhigh-precision astrometric measurements at the $0.05 \mu$ as $(1 \sigma)$ accuracy level, sufficient to detect dynamical effects due to orbiting planets of mass even lower than Earth's around the nearest stars. Such a survey mission would provide the actual planetary masses and the full orbital geometry for all the components of the detected planetary systems down to the Earth-mass limit. The NEAT performance limits can be achieved by carrying out differential astrometry between the targets and a set of suitable reference stars in the field. The NEAT instrument design consists of an off-axis parabola singlemirror telescope $(\mathrm{D}=1 \mathrm{~m})$, a detector with a large

The complete affiliations are given at the end of the paper. The full list of members of the NEAT proposal is avialable at http://neat.obs.ujf-grenoble.fr. made of 8 small movable CCDs located around a fixed central CCD, and an interferometric calibration system monitoring dynamical Young's fringes originating from metrology fibers located at the primary mirror. The mission profile is driven by the fact that the two main modules of the payload, the telescope and the focal plane, must be located $40 \mathrm{~m}$ away leading to the choice of a formation flying option as the reference mission, and of a deployable boom option as an alternative choice. The proposed mission architecture relies on the use of two satellites, of about $700 \mathrm{~kg}$ each, operating at L2 for 5 years, flying in formation and offering a capability of more than 20,000 reconfigurations. The two satellites will be launched in a stacked configuration using a Soyuz ST launch vehicle.

The NEAT primary science program will encompass an astrometric survey of our 200 closest F-, Gand K-type stellar neighbors, with an average of 50 visits each distributed over the nominal mission duration. The main survey operation will use approximately $70 \%$ of the mission lifetime. The remaining $30 \%$ of NEAT observing time might be allocated, for example, to improve the characterization of the architecture of selected planetary systems around nearby targets of specific interest (low-mass stars, young stars, etc.) discovered by
\end{abstract}


Gaia, ground-based high-precision radial-velocity surveys, and other programs. With its exquisite, surgical astrometric precision, NEAT holds the promise to provide the first thorough census for Earth-mass planets around stars in the immediate vicinity of our Sun.

Keywords Exoplanets · Planetary systems · Planetary formation · Astrometry $\cdot$ Space Mission

\section{Introduction}

Exoplanet research has grown explosively in the past decade, supported by improvements in observational techniques that have led to increasingly sensitive detection and characterization. Among many results, we have learned that planets are common, but their physical and orbital properties are much more diverse than originally thought.

A lasting challenge is the detection and characterization of planetary systems consisting in a mixed cortege of telluric and giant planets, with a special regard to telluric planets orbiting in the habitable zone (HZ) of Sun-like stars. The accomplishment of this goal requires the development of a new generation of facilities, due to the intrinsic difficulty of detecting Earth-like planets with existing instruments. The proposed NEAT mission has been designed to enter a new phase in exoplanetary science by delivering an enhanced capability of detecting small planets at and beyond $1 \mathrm{AU}$.

Astrometry is probably the oldest branch of astronomy. Greeks developed it and noticed that the position of most stars were stable in the sky, but the few that were moving became known as planets ( $\pi \lambda \alpha \dot{\alpha} \varepsilon \tau \varepsilon \varsigma$ $\dot{\alpha} \sigma \tau \varepsilon$ pes $=$ moving stars), pointing to a major difference in their nature. Thanks to the precise astrometric measurements of planet positions by Tycho Brahe in the 16th century, Johannes Kepler established that these objects were orbiting the Sun on elliptical orbits, expanding the Copernican revolution. After Hipparcos, Gaia will play an important role in finding many systems with giant planets in our Galaxy. We want to extend these revolutions with the NEAT mission, namely to discover and characterize Earth-mass planets in Earth-like orbits around stars like the Sun, by capturing infinitesimal displacements with unprecedented accuracy.

In Sect.2, we present the science objectives of NEAT, we describe the principle of the differential astrometry technique and we give a list of potential targets. In Sect. 3. after listing the technical challenges, we present the instrumental concept. We explain how to reach the performance and we give a summarized description of the payload, the mission and the spacecraft. In Sect. 4 . we discuss both astrophysical and technical issues. Recommandations by the community summarized in Sect. 5 is an incentive to pursue the development of this mission in the future.

\section{NEAT Science}

\subsection{Science objectives}

The prime goal of NEAT is to detect and characterize planetary systems orbiting bright stars in the solar neighborhood that have a planetary architecture like that of our Solar System or an alternative planetary system made of Earth mass planets. It will allow the detection around nearby stars of planets equivalent to Venus, Earth, (Mars), Jupiter, and Saturn, with orbits possibly similar to those in our Solar System. It will permit to detect and characterize the orbits and the masses of many alternate configurations, e.g. where the asteroid belt is occupied by another Earth mass planet and no Jupiter. The NEAT mission will answer the following questions:

- What are the dynamical interactions between giant and telluric planets in a large variety of systems?

- What are the detailed processes involved in planet formation as revealed by their present configuration?

- What are the distributions of architectures of planetary systems in our neighborhood up to $\approx 15 \mathrm{pc}$ ?

- What are the masses, and orbital parameters, of telluric planets that are candidates for future direct detection and spectroscopic characterization missions?

Special emphasis will be put on planets in the Habitable Zone because this is a region of prime interest for astrobiology. Indeed orbital parameters obtained with NEAT will allow spectroscopic follow-up observations to be scheduled precisely when the configuration is the most favorable.

\subsection{High-precision differential astrometry}

The principle of NEAT is to measure accurately the offset angles between a target and 6-8 distant reference stars with the aim of differentially detecting the reflex motion of the target star due to the presence of its planets. An example of a field that will be observed is shown in Fig. 1 and a simulation of what will be measured is displayed in Fig. 2.

The output of the analysis is a comprehensive determination of the mass, orbit, and ephemeris of the different planets of the multiplanetary system (namely 

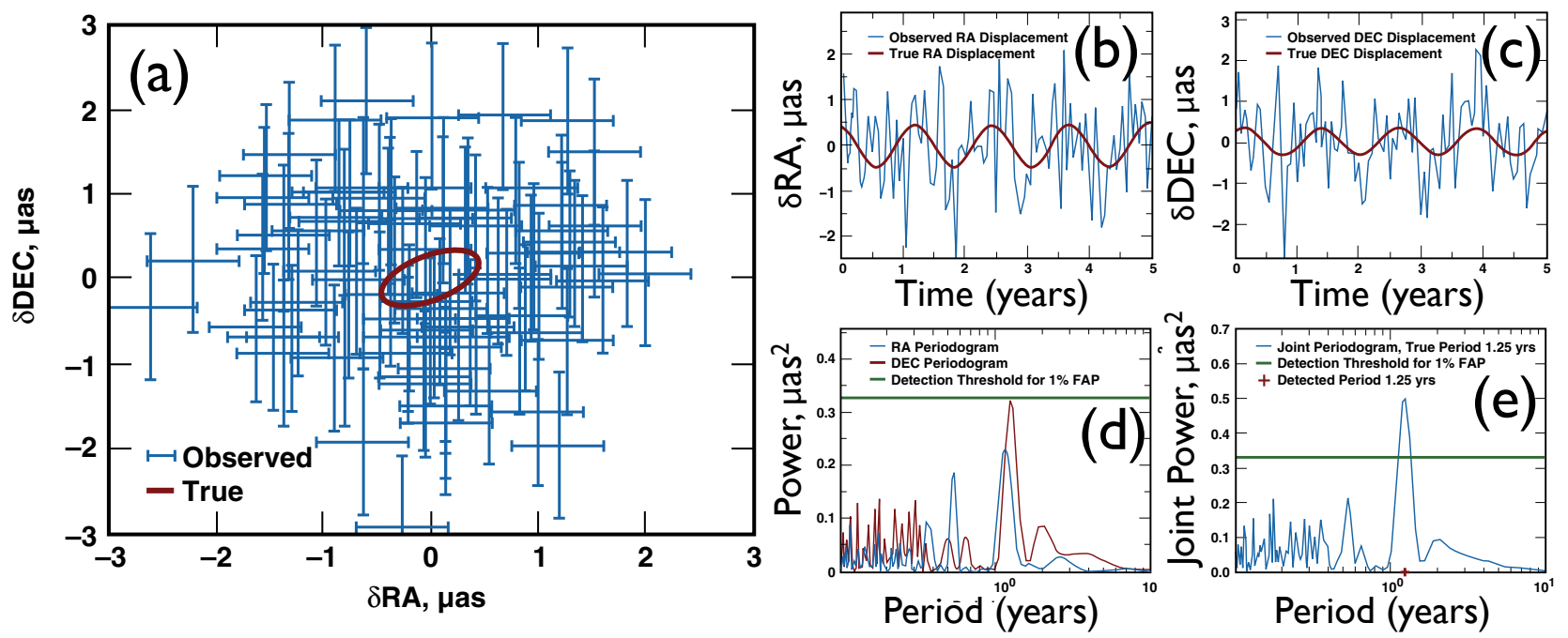

Fig. 2 Simulation of astrometric detection of a planet with 50 NEAT measurements (RA and DEC) over 5 yrs. Parameters are: $M_{P}=1.5 \mathrm{M}_{\oplus}, a=1.16 A U, M_{*}=1 \mathrm{M}_{\odot}, D=10 \mathrm{pc}, \mathrm{SNR} \geq 6$. (a) Sky plot showing the astrometric orbit (solid brown curve) and the NEAT measurements with error bars (in blue); (b) and (c) same data but shown as time series of the RA and DEC astrometric signal; (d) Separated periodogram of RA (blue line) and DEC (brown line) measurements. (e) Joint periodogram for right ascensions and declinations simultaneously. Whereas the orbit cannot be determined from the astrometric signal without the time information, its period is reliably detected in the joint periodogram $(1.25 \mathrm{yr})$ with a false-alarm probability below $1 \%$ (green line). Then, the planetary mass and orbit parameters can be determined by fitting the astrometric measurements.

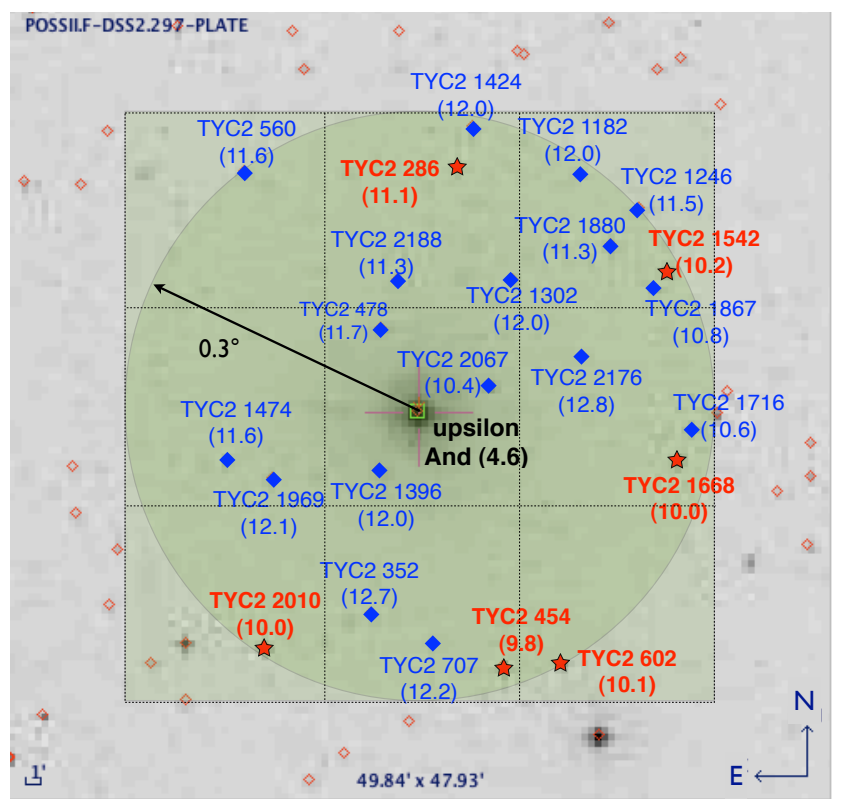

Fig. $10.3^{\circ}$ stellar field around upsilon Andromedae, a proposed NEAT target. There are six possible reference stars in this field marked in red (five $V<11$ stars and a $V=11.1$ one).

the 7 parameters $\left.M_{P}, P, T, e, i, \omega, \Omega\right)$, down to a given limit depending on the star characteristics, e.g. 0.5, 1 or $5 \mathrm{M}_{\oplus}$. The astrometric amplitude, $A$, of a $M_{*}$ mass star due to the reflex motion in presence of a $M_{P}$ mass planet orbiting around with a semi-major axis $a$ at a distance $D$ from the Sun is

$A=3\left(\frac{M_{P}}{1 \mathrm{M}_{\oplus}}\right)\left(\frac{a}{1 \mathrm{AU}}\right)\left(\frac{M_{*}}{1 \mathrm{M}_{\odot}}\right)^{-1}\left(\frac{D}{1 \mathrm{pc}}\right)^{-1} \mu$ as.

To detect such a planet, one needs to reach a precision $\sigma=A / \mathrm{SNR}$ with a typical signal-to-noise ratid ${ }^{1} \mathrm{SNR}=$ 6. If $\sigma_{0}$ is the precision that NEAT can reach in one single observation that lasts $t_{0}$ (e.g. $\sigma_{0}=0.8 \mu$ as in $t_{0}=1 \mathrm{~h}$ ), when observing the same source $N_{\text {visits }}$ times during $T_{\text {visit }}$ each visit requires

$T_{\text {visit }}=t_{0}\left(\frac{A}{\operatorname{SNR} \sigma_{0}}\right)^{-2}\left(2 N_{\text {visits }}-m\right)^{1 / 2}$

for a given $N_{\text {visits }}$, and with $m=5+7 p$ parameters where $p$ is the number of planets in the system since there are 5 parameters characterizing the star astrometric motion and 7 parameters for each orbit. $N_{\text {visits }} \approx 50$ is sufficient to solve for the parameters of 3 to 5 planets per system, for a 5-yr duration of the mission.

\subsection{Targets}

A possible target list is shown in Table 1 where we consider the list of the nearest F, G, K stars deduced from the Hipparcos 2007 catalogue (new data reduction

1 Simulations like the ones presented in Fig. 2 show that $\mathrm{SNR}=5.8$ results in a false alarm probability of $1 \%$. 
Table 1 Partial list of possible targets, the full list is available on the NEAT website (http://neat.obs.ujf-grenoble. fr/ $I M G / x l s /$ Proposal_targets_total. $x l s$ ). Stars are ranked by decreasing astrometric signal for a planet in its habitable zone (HZ). This signal $A(\mu \mathrm{as})$ is calculated for $0.5,1$ and $5 \mathrm{M}_{\oplus}$ planets around the 5,70 and 200 first stars, respectively, assuming that the planet is located at the inner boundary of the HZ that secures its detection whenever the planet is in this zone. The corresponding integration time $\left(t_{\mathrm{visit}}\right.$ in $\left.\mathrm{h}\right)$ and cumulated times $\left(t_{\mathrm{tot}}\right.$ in $\left.\mathrm{h}\right)$ are calculated for a detection with an equivalent $\mathrm{SNR}=6$. The total time corresponds to $70 \%$ of the available mission time with a $22 \%$ margin.

\begin{tabular}{|c|c|c|c|c|c|c|c|c|c|c|c|c|c|c|}
\hline \multirow[t]{2}{*}{ Rank } & \multirow[t]{2}{*}{ Star_ident. } & \multirow[t]{2}{*}{ Name } & \multirow[t]{2}{*}{ Vmag } & \multirow[t]{2}{*}{ SpType } & \multirow[t]{2}{*}{$D(p c)$} & \multicolumn{2}{|c|}{$\log R^{\prime} H K H Z_{\text {in }}(A U)$} & \multirow[t]{2}{*}{$t_{\text {tot }}(h)$} & \multirow{2}{*}{$\begin{array}{l}A \text { (uas) } \\
\text { limit }=0,5\end{array}$} & \multicolumn{5}{|c|}{$t_{\text {visit }}(h), A$ (uas) $t_{\text {visit }}(h) ; A$ (uas) (visit $(h)$} \\
\hline & & & & & & & & & & & 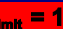 & & $=5$ & \\
\hline 1 & HIP16537 & eps Eri & 3.72 & $\mathrm{~K} 2 \mathrm{~V}$ & 3,2 & $-4,620$ & 0,57 & 139 & 0,35 & 2,5 & & & & \\
\hline 2 & HIP8102 & LHS 146 & 3.49 & G8V & 3,7 & $-4,958$ & 0,58 & 337 & 0,29 & 3,7 & & & & \\
\hline$(\ldots)$ & & & & & & & & - & - & - & & & & \\
\hline 4 & HIP104214 & 61 Cyg A & 5,20 & K5V & 3,5 & $-4,764$ & 0,34 & 804 & 0,25 & 4,8 & & & & \\
\hline 5 & HIP19849 & 40 Eri & 4.43 & K1V & 5,0 & $-5,380$ & 0,62 & 1094 & 0,24 & 5,6 & & & & \\
\hline 7 & HIP99240 & del Pav & 3.55 & G5IV & 6,1 & $-5,000$ & 0,96 & 1265 & & & 0,45 & 1,5 & & \\
\hline 8 & HIP96100 & sig Dra & 4.67 & KOV & 5,8 & $-4,832$ & 0,63 & 1357 & & & 0,44 & 1,6 & & \\
\hline$(\ldots)$ & & & & & & & & - & & & - & - & & \\
\hline 69 & HIP64797 & LTT 13852 & 6,49 & $\mathrm{~K} 2 \mathrm{~V}$ & 11,1 & $-4,630$ & 0,56 & 16957 & & & 0,20 & 7,5 & & \\
\hline 70 & HIP23835 & LHS 1736 & 4.91 & G4V & 15,4 & $-5,127$ & 1,26 & 17349 & & & 0,20 & 7,6 & & \\
\hline 71 & HIP47592 & LTT 3558 & 4.93 & GOV & 15,0 & $-4,862$ & 1,18 & 17377 & & & & & 1,00 & 0,31 \\
\hline 72 & HIP26779 & V538 Aur & 6.21 & K1V & 12,3 & $-4,550$ & 0,68 & 17405 & & & & & 1,00 & 0,31 \\
\hline$(\ldots)$ & & & & & & & & $\begin{array}{l}- \\
-\end{array}$ & & & & & - & - \\
\hline 199 & HIP79537 & LHS 413 & 7,53 & KOV & 12,9 & $-5,205$ & 0,41 & 22013 & & & & & 0,70 & 0,64 \\
\hline 200 & HIP18859 & HD 25457 & 5.38 & F5V & 18,8 & $-4,600$ & 1,02 & 22058 & & & & & 0,69 & 0,65 \\
\hline
\end{tabular}

Table 2 Left: summary of the main program capabilities and required resources. Right: Time and allocated maneuvers for the different programs: (1) the Gaia Mission and its Exoplanet Science Potential; (2) NEAT follow-up program of Gaia detected planetary systems; (3) observations of young stars; and (4) characterizing planetary systems around some of the closest $M$ stars.

\begin{tabular}{|c|c|c|c|c|c|c|}
\hline \multirow{3}{*}{$\begin{array}{l}\text { Number } \\
\text { of stars }\end{array}$} & \multirow{3}{*}{$\begin{array}{c}\text { Mass } \\
\text { threshold } \\
(\mathbf{M} \oplus)\end{array}$} & \multirow{3}{*}{$\begin{array}{c}\text { Cumulated } \\
\text { time } \\
\text { (h) }\end{array}$} & \multirow{3}{*}{$\begin{array}{l}\text { Number } \\
\text { of visits }\end{array}$} & Program & \multirow{2}{*}{$\begin{array}{c}\text { Time (h) } \\
3,650\end{array}$} & \multirow{2}{*}{ Maneuver } \\
\hline & & & & Transfert + com. & & \\
\hline & & & & Main & 22,100 & 10,000 \\
\hline 5 & 0.5 & 1,100 & 500 & Add. I-3 & 5,500 & 2,000 \\
\hline 70 & 1 & 15,600 & 3,500 & Add.4 & 2,750 & 1,000 \\
\hline 200 & 5 & 6,400 & 6,000 & total margins & $9,800(22 \%)$ & 7,000 \\
\hline \multicolumn{2}{|c|}{ Total } & 22,100 & 10,000 & Total & 43,800 h (5 yrs) & 20,000 \\
\hline
\end{tabular}

by van Leeuwen, 2007), disregarding spectroscopic binaries, and stars with an activity level 5 times greater than that of the Sun because of their astrometric noise (only $4 \%$ of the sample, Lagrange et al. 2011) and for which we compute the astrometric signal for a planet with given mass in the HZ of the stars (Kaltenegger et al. 2010). Conservatively, we select the inner part of the $\mathrm{HZ}$ in order to be able to detect the planet whatever is its location in the HZ. The required number of visits and cumulative time to observe this list of target stars is summarized in Table 2. The list corresponds to an exhaustive search for 1 Earth mass planets (resp. 5 Earth mass planets) around K stars up to 6 pc (resp. 12 pc), G stars up to 10 pc (resp. 17 pc), and F stars up to 14 pc (resp. 19 pc) in the whole HZ of the star, excluding spectroscopic binaries and very active stars. The spatial repartition of targets is shown in Fig. 3. $60 \%$ of the NEAT targets (118) are brighter than $V=6$ and therefore will not be investigated by Gaia because of its bright limit. So, even if some of those sources do not harbor Earth-like planets, NEAT will be contributing

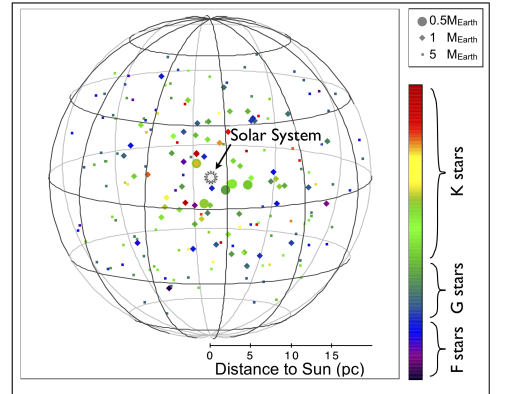

Fig. 3 Representation of the NEAT targets in the 3D sphere of our neighborhood ( $D$ up to $\approx 15 \mathrm{pc}$ ). They correspond to a volume limited sample of all stars with spectral types between $\mathrm{F}$ and $\mathrm{K}$.

to the improvement of our knowledge about the neighborhood of our Solar System. In that respect, NEAT observations will not only be complementary to Gaia's ones, but NEAT data will also form a base to improve Gaia results.

In addition to the survey for the NEAT main science program, we propose that $30 \%$ of NEAT time is allocated to study some objects of interest (planets around M dwarfs, young stars, multiple systems,... discovered by Gaia and others). The global required amount of time and number of maneuvers is listed in Table2 (right part).

\section{NEAT concept}

Our goal is to detect the signal corresponding to the reflex motion of a Sun-like star at 10 pc due to an Earthmass planet in its HZ, with an equivalent final SNR of 6 . 
That astrometric signal is $0.30 \mu$ as. The required noise floor is $0.05 \mu$ as, over 100 times lower than Gaia's best precision ( $7 \mu$ as).

\subsection{Technical challenges}

Achieving sub-micro-arcsecond astrometric precision, e.g. $0.8 \mu$ as, in 1 hour and a noise floor under $0.05 \mu$ as with a telescope of diameter $\mathrm{D}$ requires mastering all effects that could impact the determination of the position of the point spread function. The typical diffraction limited size of an unresolved star is about $1.2 \lambda / D$, which corresponds to 0.16 arcseconds for a 1-m telescope operating in the visible spectral region. The challenge is therefore to control these systematics effects to a level better than 1 part in 3 million $\left(1: 3 \times 10^{6}\right)$. Even though differential astrometry of stars within the same field of view softens somewhat the requirement, this level of accuracy can only be obtained in an atmosphere-free space environment.

Sub-micro-arcsecond level astrometry requires solutions to four challenges:

- Photon noise. Most targets are $R \leq 6$ mag stars, but the required reference stars are $R \leq 11 \mathrm{mag}$ so they dominate the photon noise. Using the mean stellar density in the sky, one finds that a field of view (FOV) as large as diam $0.6^{\circ}$ is needed to get several (6 to 8) of theses references (see e.g. Fig 1).

- Beam walk. A classical three mirror anastigmat (TMA) telescope can also manage a $0.6^{\circ}$ diffraction limited FOV. However the light coming from different stars, and therefore from different directions, will hit the secondary and tertiary mirrors on different physical parts of the mirrors. The mirror defects will therefore produce different and prohibitive astrometric errors between the images of the stars. Using a single mirror telescope solves this problem. To obtain sufficiently high angular resolution, a long focal length $(\approx 40 \mathrm{~m})$ for this mirror is needed, with no intermediate mirrors, a relatively unusual solution in modern optical astronomy.

- Stability of the focal plane. Proper Nyquist sampling with typical detector pixels of the order of $10 \mu \mathrm{m}$ requires a focal plane at a focal length of $40 \mathrm{~m}$. Such a focal plane covering a FOV of $0.6^{\circ}$ diameter would yield a costly detector mosaic with $40,000 \times 40,000 \approx 10^{9}$ pixels. Sub-microarcesc astrometry over a $0.6^{\circ}$-diameter FOV requires the geometry of the focal plane to be stable to $\approx 1$ : $2 \times 10^{-10}$. Therefore thermal stability of the focal plane geometry will be a major challenge although it has to be investigated in details. Instead of building

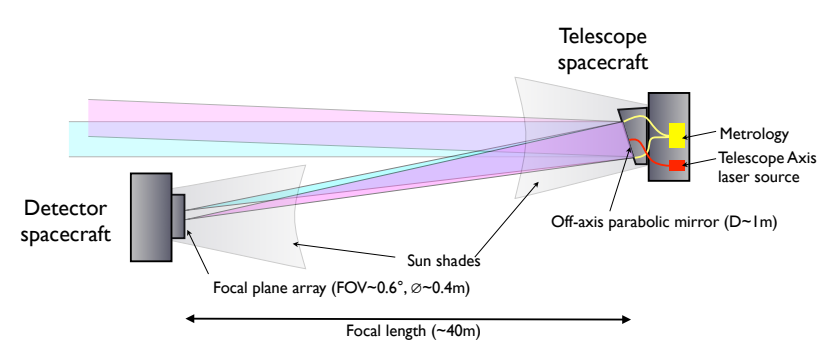

Fig. 4 Proposed concept for a very high precision astrometry mission. It consists in two separated modules, the first one carrying the primary mirror (upper right) and the second one the detector plane (bottom left).

a gigapixel focal plane with unprecedented stability we plan to use 9 small $512 \times 512$ CCDs (Fig. 5) and a laser metrology system to measure the position of every pixel to the required precision, once every 10 to $30 \mathrm{~s}$. We do not rely on their positioning, but measure it accurately with a laser metrology based on dynamic interference fringes.

- Quantum efficiency (QE) variations. The dynamic fringes also allow the measurement of the inter- and intra-pixel QE variations. We characterize each pixel response with six parameters such that the systematic errors are kept below $10^{-6}$. This is a process derived from the SIM studies.

These effects have in the past hampered the performance of space missions like HST.

\subsection{Instrumental concept}

The proposed mission is based on a concept recently proposed by M. Shao and his colleagues that results from the experience gained in working with many astrometry concepts (SIM, SIM-Lite, corono-astrometry 2). The concept is sketched in Fig. 4 and consists of a primary mirror - an off-axis parabolic 1-m mirror- a focal plane $40 \mathrm{~m}$ away, and metrology calibration sources. The large distance between the primary optical surface and the focal plane can be implemented as two spacecraft flying in formation, or a long deployed boom. The focal plane with the detectors having a field of view of $0.6^{\circ}$ is shown in Fig. 5 . It has a geometrical extent of $0.4 \mathrm{~m} \times 0.4 \mathrm{~m}$. The focal plane is composed of eight $512 \times 512$ visible CCDs located each one on an XY translation stage while the central two CCDs are fixed in position. The CCD pixels are $10 \mu \mathrm{m}$ in size.

The principle of the measurement is to point the spacecraft so that the target star, which is usually brighter $(R \leq 6)$ than the reference stars $(R \leq 11)$, is located on the axis of the telescope and at the center of the central

2 See Guyon et al. (2010) 

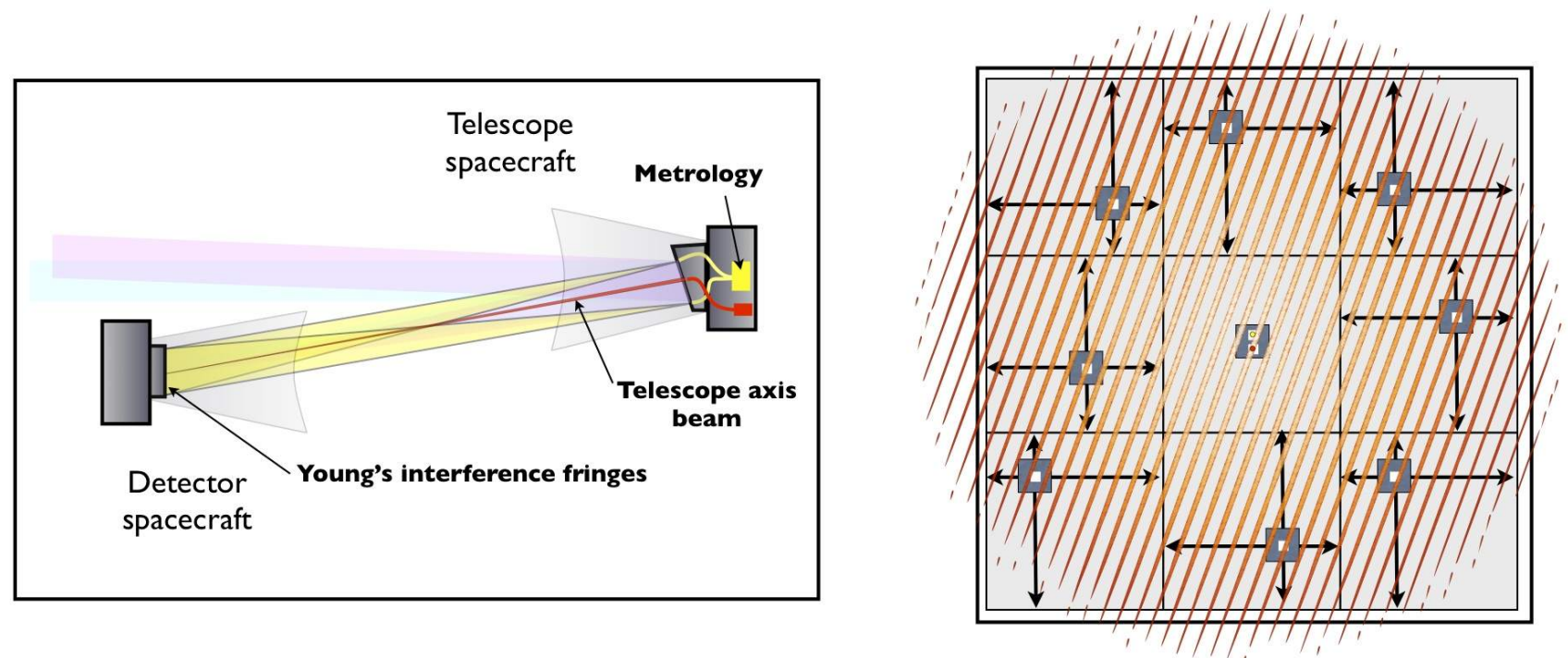

Fig. 6 Principle of the metrology and the axis tracker. Left panel: the metrology laser light (in yellow) is launched from fibers located at the edge of the mirrors. Right panel: the laser beams interfere over the detector plane. Only the fringes corresponding to a pair of fibers are represented on this figure and they are not to scale, since the fringe spacing is equal to the PSF width. The axis tracker (sketched in red on the left panel) is a laser beam launched in the center of the mirror that is monitored in the lower central CCD.

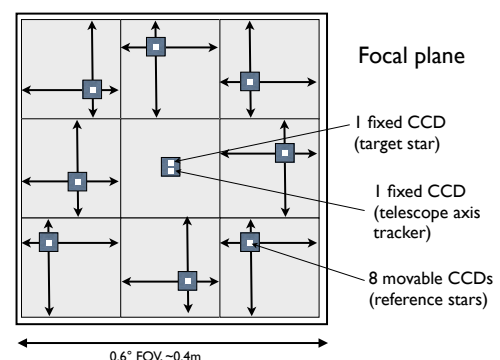

Fig. 5 Schematic layout of the focal plane. The field of view is divided in $3 \times 3$ sub-fields. Exterior subfields have visible arrays which can be moved in $\mathrm{X}$ and $\mathrm{Y}$ directions to image the reference stars. The central field has two fixed arrays, one for the target star and one for the telescope axis tracker.

CCD. Then the 8 other CCDs are moved to center each of the reference stars on one of them. To measure the distance between the stars, we use a metrology calibration system that is launched from the telescope spacecraft and that feeds several optical fibers (4 or more) located at the edge of the mirror. The fibers illuminate the focal plane and form Young's fringes detected simultaneously by each CCD (Fig. 6). The fringes have their optical wavelengths modulated by acoustic optical modulators (AOMs) that are accurately shifted by $10 \mathrm{~Hz}$, from one fiber to the other so that fringes move over the CCDs. These fringes allow us to solve for the XYZ position of each CCD. An additional benefit from the dynamic fringes on the CCDs is to measure the $\mathrm{QE}$ of the pixels (inter-, and intra-pixel dependence). The
CCDs are read at $50 \mathrm{~Hz}$ providing many frames that will yield high accuracy.

With the proposed concept, it is possible to achieve all of the main technical requirements:

- Focal plane stability. Instead of maintaining a focal plane geometry stable at the $0.1 \mathrm{~nm}$ level for a 5-yr duration, which is impossible, we implement a metrology for every pixel at the sub-nanometer level, with an interferometric system that has been qualified by the SIM-Lite laboratory demonstrators.

- Reference frame. By measuring the fringes at the sub-nanometer level using the information from all the pixels of each CCD (SIM-Lite technology), it is possible to solve for the position of all reference stars compared to the central target with an accuracy of $0.8 \mu$ as per hour. The field of view of $0.6^{\circ}$ allows us to have 6 to 8 reference stars brighter than $V=11$ in most fields.

- Photon noise. The field of $0.6^{\circ}$ provides about 6 to 8 stars of magnitude brighter than $R=11$. The number of photons received by one 11-mag star on the system is $\approx 4.1 \times 10^{9} \mathrm{ph} / \mathrm{hr}$. Since the FWHM of diffraction-limited stars is $1.2 \lambda / D=0.16$ arcsecond, the photon noise limit in $1 \mathrm{~h}$ of integration due to a set of 6 reference stars is $(\lambda / 2 D) / \sqrt{6 N} \approx 0.5 \mu$ as. With more than $50 \times 2$ measurements of a few hours spread over $5 \mathrm{yrs}$, the equivalent precision is $0.05 \mu \mathrm{as}$ in RA and Dec, corresponding to the detection of the $0.30 \mu$ as signal with a SNR $\approx 6$. 
- Large-scale calibration. The detector plane does not have to be fully covered by pixels, since the positions of the reference stars are known from available catalogues (10-20 mas for Tycho 2, and about tens of $\mu$ as for Gaia). For the target stars $(R \leq 6)$, we use the Hipparcos catalog (few mas accuracy). This corresponds to $<1 / 10^{\text {th }}$ of the PSF or the fringe width. The number of fringes between the target star and the reference stars is then known, only the positions of the star centroids relative to the interferometric fringe have to be measured accurately.

The use of 10 small CCDs drastically reduces the cost of what would otherwise be a giga-pixel focal plane and also helps to control systematics. With such a concept, the mission performance would be similar to, and even more favorable for exoplanets, than what was proposed for SIM-Lite with 5 years of operation, but at the price of giving up all-sky astrometry and the corresponding science objectives.

\subsection{Performance assessment and error budget}

Achieving a relative precision of $2 \times 10^{-10}$ is slightly better than the precision achievable by only the combination of our metrology laser, the thermal expansion coefficient of the primary mirror and our expected temperature stability. Achieving our target precision relies on not only the metrology stability, but also on the precise knowledge of the positions of the multiple reference stars used since the expected motions of the references cannot be considered as fixed (see discussion in Sect. 4.1). Our comprehensive error budget takes into account all sources of error, including instrumental effects, photon noise and astrophysical errors in the reference star positions.

The biggest term is the brightness dependent error for the set of Reference stars. The half-width of the PSF for the coma-aberrated images of the reference stars is about $19 \mu \mathrm{m}$ on the focal plane (or $100 \mathrm{mas}$ on the sky). After $1 \mathrm{~s}$ of integration, $1.3 \times 10^{6}$ photoelectrons are detected for each of the 11-mag reference star; their centroid location can be estimated to $0.016 \mu \mathrm{m} \mathrm{rms}\left(1.6 \times 10^{-3}\right.$ pixel or $\left.0.08 \mathrm{mas}\right)$. Since all the stars are measured simultaneously, the stars do not need to be kept centered on the detector at the submas level, but only to a fraction of the PSF width to avoid spreading of the photon outside of the PSF and therefore cause the PSF effective width to be larger. A tenth of pixel $(1 \mu \mathrm{m})$ stability over the one-second integration is sufficient. After $3400 \mathrm{~s}$ of integration, the statistical averaged position of the barycenter of the set of reference stars ( $R \leq 11 \mathrm{mag}$ ) will be measured with a residual $0.126 \mathrm{~nm}(0.63 \mu \mathrm{as})$ uncertainty. Similarly, the position of the target star $(R \leq 16 \mathrm{mag})$ will be measured with a residual $0.024 \mathrm{~nm}(0.12 \mu \mathrm{as})$ uncertainty. Although the spacecraft will have moved by several arcseconds, the differential position between the target star and the barycenter of the set of reference stars will be determined to $0.64 \mu$ as.

Similarly, the focal plane metrology system will have determined the differential motion of the target CCD relative to the barycenter of the set of reference CCDs with an error smaller than $0.16 \mu$ as after $60 \times 1$ s metrology measurements.

NEAT will not be capable of measuring the absolute separation between the target and the set of reference stars to $0.8 \mu$ as. NEAT objectives will therefore be to measure the change in the relative position of those stars between successive observations spread over the mission life, with an error of $0.8 \mu$ as for each one hour visit. The six major errors terms are captured in the simplified version of the error budget shown in Fig. 7.

If unmonitored, the displacement of the projected field aberrations on the focal plane would produce a $60 \mu$ as differential astrometric error per arcsecond of relative spacecraft motion. The telescope axis tracker will monitor the relative position of the focal plane relative to the parabola axis simultaneously with the stellar observation with a 1 mas accuracy per hour. This will be sufficient to correct the observations during postprocessing for the field-dependent aberration to better than $0.1 \mu$ as.

Static figure errors of the primary mirror will produce centroid offsets that are mostly common-mode across the entire field of view. Differential centroid offsets are significantly smaller than the field-dependent coma and are in fact negligible. Similarly, changes in the primary mirror surface error, e.g. due to thermal dilatation ${ }^{3}$ meteorite impacts,... produce mostly commonmode centroid shifts and negligible differential centroid offsets. On the other hand, displacement and changes in the shape of the PSF would couple with the CCD response if the CCD response is not properly calibrated. This is continuously done by the metrology fringes.

\subsection{Design of the payload subsystems}

Focal plane assembly. A proposition for implementation of the focal plane is shown in Fig. 8. The detector is foreseen to be a CCD fabricated by the E2V technologies company in UK. The target star, the reference

3 The coefficient of thermal expansion of the mirror is about 100 times smaller than those of the elements that compose the detector. The metrology parameters are constantly monitored. 


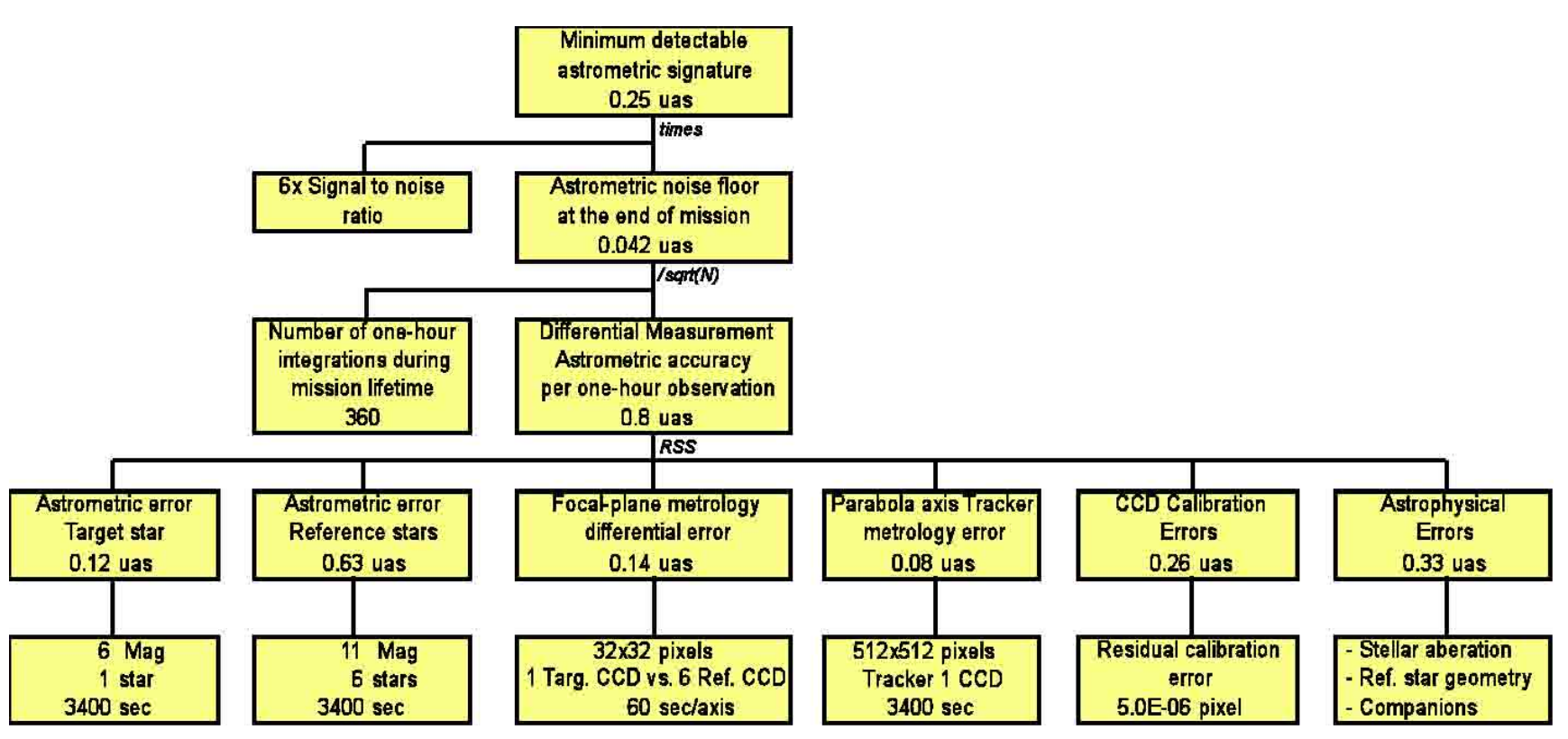

Fig. 7 Top-level error budget for NEAT. It shows how the $0.8 \mu$ as accuracy enables the detection of $0.3 \mu$ as signatures with a signal to noise of 6 after $360 \mathrm{~h}$ of observation. It also shows the major contributors to the astrometric error.
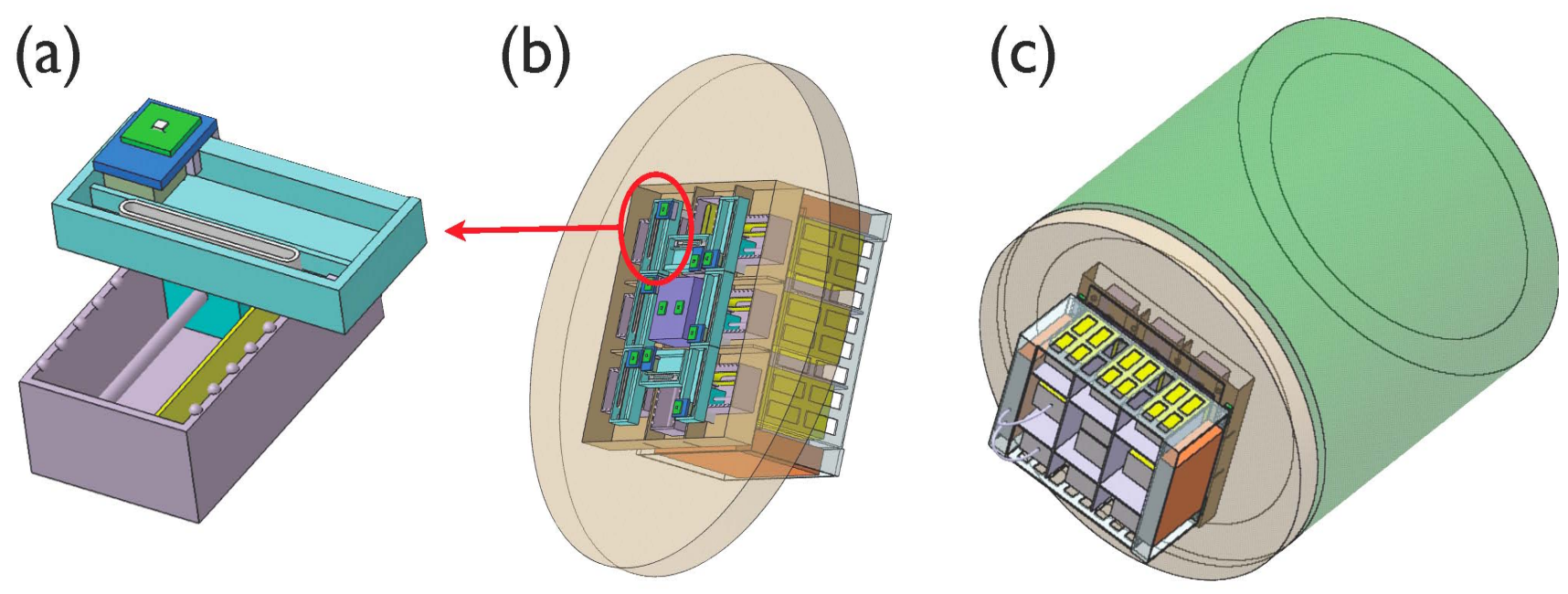

Fig. 8 Views of the focal plane assembly. (a) Magnified view of one of the 8 XY translation stages of the focal plane. In yellow, the $512 \times 512 \mathrm{CCD}$ and its support. In blue and green the two translation stages. (b) The front part of the focal plane with its 8 movable CCDs and two fixed CCDs at the center. (c) The electronics racks.

star and the telescope axis tracker will all use the same CCD that includes the capability to read windowed images, typically $10 \times 10$ to $30 \times 30$ pixels. The $8 \mathrm{XY}$ tables consist of two linear tables mounted on top of each other. Each table uses a piezo-reptation motor 4 . a linear ball bearing system and an optical incremental encoder. These motors fulfill several requirements of simplicity: they are self-locked when they are not pow-

\footnotetext{
4 Such reptile motors have been qualified by the Swiss firm RUAG for the LISA GPRM experiment.
}

ered; they can be used both for large displacements by stepping up to $100 \mathrm{~mm} \times 100 \mathrm{~mm}$ and elementary ana$\log$ motion down to $50 \mathrm{~nm}$. Since 8 tables are used in parallel in the focal plane, the loss of one table is not a single point failure. An alternative implementation could be to drive the XY tables with ball screws and rotary motors. The limited resolution of such a motor stage (about $5 \mu \mathrm{m}$ ) could be supplemented by a second high-resolution piezo XY table mounted on top of the

\footnotetext{
5 such as the Cedrat XY25XS
} 


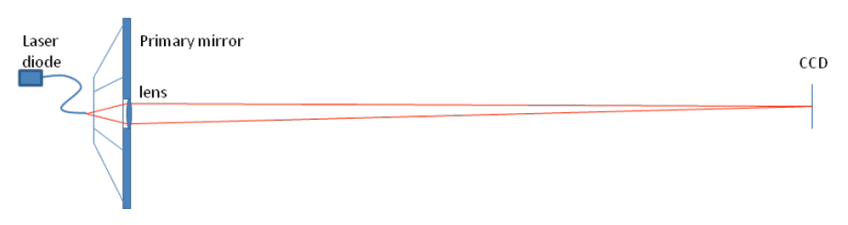

Fig. 9 Laser metrology of the telescope axis tracker. The launcher embedded in the primary mirror substrate forms a $600 \mu \mathrm{m}$ (60 pixels) FWHM diffraction limited image of the fiber output on the Telescope Axis Tracker CCD. By sampling the entire $512 \times 512$ pixel detector, the parabola axis can be tracked over a 26 arcsec range, with a 0.3 mas estimation error at $10 \mathrm{~Hz}$.

first XY table. The main structure of the focal XY tables consists of a large lightweight aluminum cylinder which is thermally controlled and in which pockets are machined for the fixation of the XY tables.

Telescope. The primary mirror is an off-axis paraboloid with a $1 \mathrm{~m}$ diameter clear aperture, an off-axis distance of $1 \mathrm{~m}$ and a focal length of $40 \mathrm{~m}$. It would be fabricated in either Zerodur or ULE, 70\% light-weighted and weight about $60 \mathrm{~kg}$. The surface quality should be better than $\lambda / 4$ peak-to-valley and would be coated with protected aluminum. A $50 \mathrm{~mm}$ hole at the center of the mirror accommodates the beam launcher for the telescope axis tracker. The three bipods on the back of the mirror support the mirror with minimum deflection. The bipods interface with the tip-tilt stage made of 3 preloaded piezo stacks on parallel flexures that provide the $+/-6$ arcsecond amplitude for two-axis articulations. The entire primary mirror assembly interface to the telescope payload plate is a $34 \mathrm{~kg}$ hogged-out aluminum plate. This plate also hosts the metrology source, the telescope drive electronics, the telescope baffle and the interface to the spacecraft. The telescope axis tracker is used to estimate the location of the primary mirror axis with respect to the focal plane in order to monitor it and then correct for the telescope field dependent errors. The sensor is the second fixed CCD located in the focal-plane. The launcher would consist of either an achromatic doublet or an aspherical singlet lens, embedded in the primary mirror substrate at its center and a single-mode fiber-coupled laser diode. Fig. 9 shows how the fiber tip is re-imaged onto telescope axis tracker CCD in the focal plane.

Laser metrology. The focal-plane metrology system consists of the metrology source similar to the one developed for SIM (Erlig et al., 2010), the metrology fiber launchers and the focal plane detectors (CCDs) which alternatively measure the stellar signal (57 s observations) and the metrology (1s per axis every minutes). The metrology fiber launchers consist of nominally four optical fibers attached to the primary mirror substrate. Three of them are located around the edge of the mirror, and are used by pairs in order to conduct three redundant measurements of the relative location of the CCDs. The fourth fiber is located inside the clear aperture, and is used in combination with each of the three other fibers to produce three additional measurements during focal plane calibration and calibrate the distance mirror-focal plane.

Pointing servo systems. The pointing of the telescope from one target to the next one is accomplished by the two spacecraft in formation flying. The target stars will be typically separated by $10^{\circ}$. Re-pointing of the telescope will require rotation of the two spacecraft by several degrees using reaction wheels and translation of the telescope spacecraft by several meters using hydrazine propulsion. Fine positioning of the focal plane relative to the mirror is done by cold gas propulsion system, and at the end of the maneuver, the telescope spacecraft will be oriented to better than 3 arcseconds from the target star line of sight using star trackers and the focal plane spacecraft will be positioned to better than $2 \mathrm{~mm}$ from the primary mirror focus. At that point, the spacecraft will maintain their relative position to better $\pm 2 \mathrm{~mm}$ in shear and in separation for the duration of the observation. The separation does not require a servo-loop of the payload, because its effect is only a degradation in performance (when FWHM increases, final precision decreases in same proportion) and is managed in the error budget.

During the observation, the instrument uses a tiptilt stage behind the primary mirror to center the target star on the $32 \times 32$ pixel sub-window on the target star CCD. Once in the $32 \times 32$ pixel sub-frame mode, the target star CCD is read at $500 \mathrm{~Hz}$, and feedback control between the CCD and the tip-tilt stage can be used to keep the star centered on the detector to better than 5 milli-arc-second RMS (0.1 pixel RMS) for the duration of the observation. This is the only active feedback loop in the instrument system working at $50 \mathrm{~Hz}$; the other degrees of freedom (focal plane tip, tilt, clocking and focal-plane-to-mirror separation) are monitored but not corrected for in real-time. Prior to acquisition, the reference star CCDs will be pre-positioned to the expected location of the reference stars using the translation stages. The XY translation stage fine motion of the reference star CCDs at a $0.2 \mu \mathrm{m}$ precision enables centering of the reference stars on the detectors to better than a tenth of a pixel. Once the reference stars are acquired, the translations stages are locked for the duration of the observation. 


\subsection{Mission requirements}

The objectives of the NEAT mission require to perform acquisitions over a large number of targets during the mission timeline, associated to a $40 \mathrm{~m}$ focal length telescope satellite. The preliminary assessment of the NEAT mission requirements allows to identify the following main spacecraft design drivers.

Launch configuration and mission orbit. The L2 orbit is the preferred orbit, as it allows best formation flying performance and is particularly smooth in terms of environment. The Soyuz launch, proposed as a reference for medium class missions, offers satisfying performance both in terms of mass and volume.

Formation Flying and $\mathbf{4 0} \mathrm{m}$ focal length. The mission relies on a $40 \mathrm{~m}$ focal length telescope, for which the preferred solution is to use two satellites in formation flying. The performance to be provided by the two satellites in order to initialize the payload metrology systems are of the order of magnitude of $\pm 2 \mathrm{~mm}$ in relative motion, and of 3 arcseconds in relative pointing, which are typically compatible with Formation Flying Units and gyroless AOCS $\sqrt{6}$ architecture. In addition, at L2, the solar pressure is the main disturbance for formation flying control. As a result, surface-to-mass ratio ( $\mathrm{S} / \mathrm{M}$ ratio) is the main satellite drift contributor and should be as close as possible for the two satellites. Although satellite design can cope with these requirements, the S/M ratio of the satellites will evolve during the mission (because of fuel losses and sun angle). However, the preliminary mission assessment tends to demonstrate that the $\mathrm{S} / \mathrm{M}$ difference between the two satellites can be reduced down to $20-30 \%$, which is deemed compatible with mission formation flying requirements.

Number of acquisitions and Mission $\Delta V$. The mission aims at a complete survey of a large number of targets and the maximization of the number of acquisitions will be a main objective of the next mission phases. The mission objectives require a threshold of 20,000 acquisitions (see Sect. 3.6 for details). In addition, the time allocation for these reconfiguration maneuvers is quite limited, in order to free more than $85 \%$ of mission duration for observations. As a result, the mission is characterized by a large $\Delta V(550$ to $880 \mathrm{~m} / \mathrm{s})$ dedicated to reconfigurations, plus allocations for fine relative motion initialization and control using the $\mu$ propulsion system. This large number of reconfigurations is also driving the number of thruster firing, which are qualified to typical numbers of up to 5,000 to 50,000 with cycling as required for NEAT.

\footnotetext{
6 Attitude and Orbit Control System
}

Baffles and Parasitic Light. The mission performance relies on the ability of the focal plane to receive only star flux reflected by the telescope satellite. A first requirement is to implement baffles on the two satellites, coupled by a diaphragm on the focal plane. In addition, all parasitic light coming from telescope satellite reflections should be avoided, thus requiring all bus elements to be shielded by a black cover. Following this preliminary satellite requirement analysis, a first simple and robust mission concept has been identified.

\subsection{Preliminary Spacecraft Design}

The preliminary NEAT mission assessment allowed to identify a safe and robust mission architecture (Fig. 10, relying on high technology-readiness-level (TRL) technologies, and leaving safe margins and mission growth potential that demonstrates the mission feasibility within the medium class mission cost cap.

System Functional Description. The proposed mission architecture relies on the use of two satellites in formation flying (FF). The two satellites are launched in a stacked configuration using a Soyuz ST launcher, and are deployed after launch in order to individually cruise to their operational Lissajous orbit. Acquisition sequences will alternate with reconfigurations, during which the Telescope Satellite will use its large hydrazine propulsion system to move around the Focal Plane Satellite and to point at any specified star. At the approach of the correct configuration, the Focal Plane Satellite will use a cold gas $\mu$-propulsion system for fine relative motion acquisition. The Focal Plane Satellite will be considered as the chief satellite regarding command and control, communications and payload handling. Communications with the L2 ground station would typically happen on a daily basis through the Focal Plane Satellite, with data relay for $\mathrm{TC} / \mathrm{TM}^{7}$ from the Telescope Satellite using the FFRF 8 units. This satellite will however be equipped with a similar communication subsystem, in order to support cruise and orbit acquisition, and to provide a secondary backup link.

Formation Flying Architecture. The formation flying will have to ensure anti-collision and safeguarding of the flight configuration, based on the successful PRISMA flight heritage. In addition, the spacecraft will typically perform 12 to 20 daily reconfigurations of less than $10^{\circ}$ of the system line of sight corresponding to $7 \mathrm{~m}$ of translation of one satellite compared to the other perpendicular to the line of sight. During these configu-

\footnotetext{
7 Telecommand / Telemetry

8 Formation Flying Radio Frequency
} 

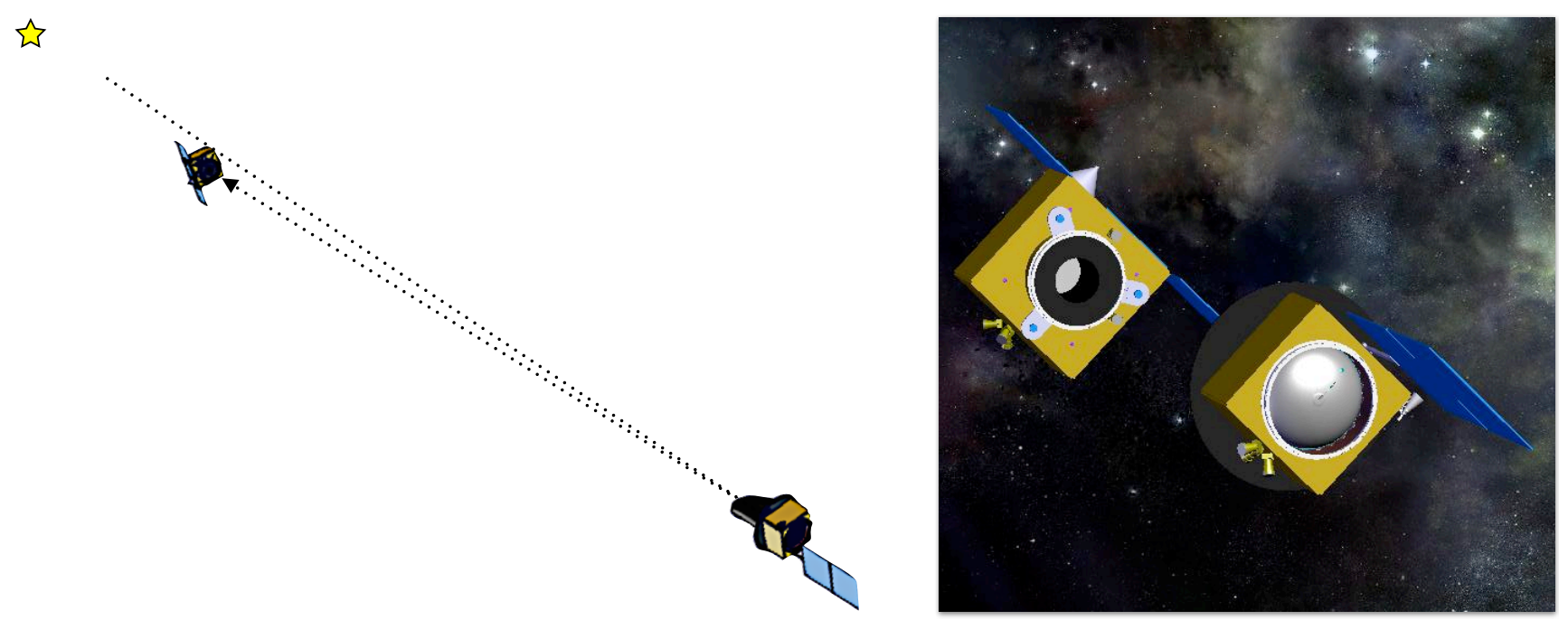

Fig. 10 Left: NEAT spacecraft in operation with the two satellites separated by $40 \mathrm{~m}$. Right: closer external view of the two satellites.

rations, the telescope satellite will perform translations - supported by the FF-RF Units - using its large hydrazine tanks $(250 \mathrm{~kg})$ for a $\Delta V \approx 605 \mathrm{~m} / \mathrm{s}$. When the two satellites will approach the required configuration, the telescope satellite will freeze, and the focal plane satellite will perform fine relative pointing control using micro-propulsion system. As a result, the micropropulsion will have to compensate for hydrazine control inaccuracies, which will require large nitrogen gas tanks $(92 \mathrm{~kg}$ for $\Delta V \approx 75 \mathrm{~m} / \mathrm{s}$ ). Finally, $28 \mathrm{~kg}$ of hydrazine carried by the FP satellite allows $\Delta V \approx 55 \mathrm{~m} / \mathrm{s}$ for station keeping and other operations.

Satellite Design Description. The design of the two satellites is based on a $1194 \mathrm{~mm}$ central tube architecture, which will allow a low structural index for the stacked configuration and provides accommodation for payloads and large hydrazine tanks. Strong heritage does exist on the two satellites avionics and AOCS. In addition, they both require similar function which would allow to introduce synergies between the two satellites for design, procurement, assembly, integration and tests. The proposed AOCS configuration is a gyroless architecture relying on reaction wheels and high-performance star trackers (Hydra Sodern), which is compatible with a 3 arcsec pointing accuracy (see end of Sect. 3.4 for payload control). The satellites communication subsystems use X-Band active pointing antenna, supported by large gain antenna for low Earth orbit positioning and cruise, coupled with a $50 \mathrm{~W}$ RF Transmitter. The active pointing medium gain antenna allows simultaneous data acquisition and downlink. A reference solution for the satellite on-board computer could rely on the Herschel-Planck avionics.

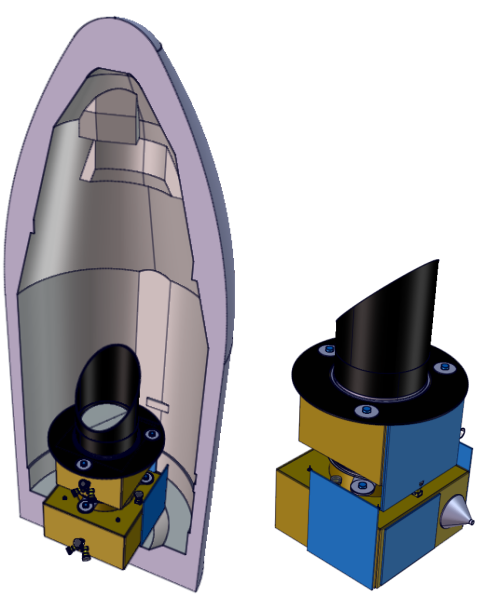

Fig. 11 NEAT stowed configuration

The two satellites would have custom mechanicalthermal-propulsion architectures. The telescope satellite features a dry mass of $724 \mathrm{~kg}$ and the focal plane satellite a dry mass of $656 \mathrm{~kg}$. The focal plane satellite carries the stacked configuration. The payload (focal plane + baffle) are assembled inside a $1194 \mathrm{~mm}$ central tube, which will also ensure the stacked configuration structural stiffness. The spacecraft bus, and large cold gas tanks, will be assembled on a structural box carried by the central tube. The proposed architecture uses a large hydrazine tank inside the $1194 \mathrm{~mm}$ central tube which offers a capacity of up to $600 \mathrm{~kg}$ hydrazine, thus allowing both a low filling ratio and a large mission growth potential. The payload module - with the payload mirror, rotating mechanisms and baffle - is then assembled on the central tube.

Proposed Procurement Approach. The NEAT mission is particularly adapted to offer a modular space- 

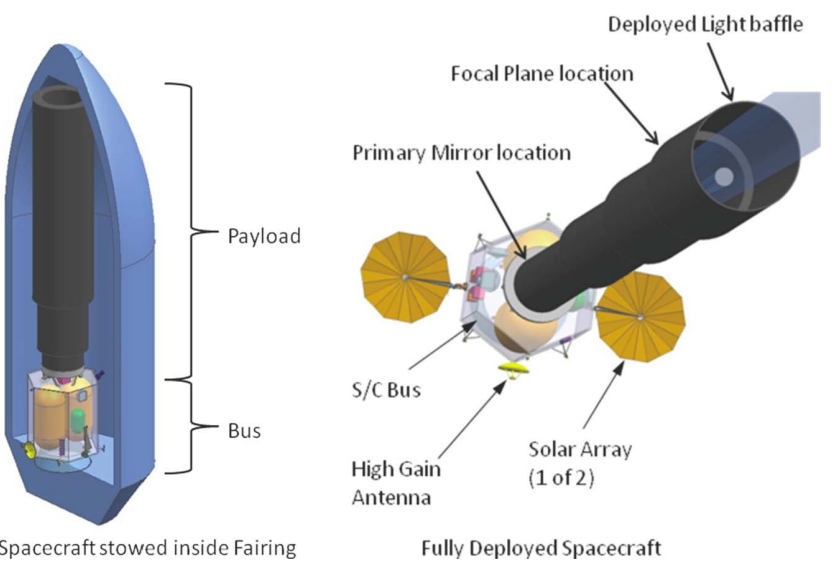

Fig. 12 Flight system concept for the deployable telescopic tube version

craft approach, with simple interfaces between payload and spacecraft bus elements. For both satellites, the payload module is clearly identified and assembled inside the structural $1194 \mathrm{~mm}$ central tube. In addition, a large number of satellite building blocks can be common to the two satellites, in order to ease mission procurement and tests. This configuration is particularly compatible with the ESA procurement scheme. The payload is made of 3 subsystems: primary mirror and its dynamic support, the focal plane with its detectors and the metrology.

Alternative mission concept. An alternative mission concept would consist of a single spacecraft with an ADAM-like 9 deployable boom (from ATK-Able engineering) that connects the telescope and the focal plane modules. The preliminary investigation made by CNES identified no show-stoppers for this option: no prohibitive oscillation modes during observation; during maneuvers, the boom oscillation modes can be excited but they can be filtered by Kalman filters (SRTM ${ }^{10}$ demonstration). The use of dampers on the boom structure allows damping at a level of $10 \%$ of the oscillations. The main worry concerns retargeting, which requires large reaction wheels or control momentum gyroscopes (CMGs) on the spacecraft due to the important inertia but propellers could be added at the boom end. A possible implementation made by JPL is shown in Fig. 12 .

\section{Discussion}

\subsection{Astrophysical issues}

Stellar activity. If all instrumental problems are controlled then the next obstacle to achieve the scientific

\footnotetext{
9 ADAM: ABLE Deployable Articulated Mast

10 Shuttle Radar Topography Mission
}

objective is of astrophysical nature, the impact of stellar activity. Spots and bright structures on the stellar surface induce astrometric, photometric and RV signals. Using the Sun as a proxy, Lagrange et al. (2011) have computed the astrometric, photometric and RV variations that would be measured from an observer located 10 pc away. It appears that the astrometric variations due to spots and bright structures are small compared to the signal of an Earth mass planet in the HZ (see also Meunier et al., 2010, Makarov et al., 2009, 2010). This remains true throughout the entire solar cycle. If we consider a star 5 times more active than the active Sun, an Earth-mass planet would still be detectable even during the highest activity phases. Such activity, or lower, translates in terms of activity index $\log \left(R_{H K}^{\prime}\right) \leq-4.35$. Consequently, in our target list, we have kept only stars with such an index (only $4 \%$ were discarded), for which their intrinsic activity should not prevent the detection of an Earth-mass planet, even during its high activity period.

Perturbations from reference stars. The vast majority of the reference stars will be $\mathrm{K}$ giants at a distance of $\approx 1 \mathrm{kpc}$. The important parameters in addition to the position are the proper motion with typical value of $\approx 1 \mathrm{mas} / \mathrm{yr}$ and the parallax whose typical value is $\approx 1$ mas. They are to be compared to the accuracy of the cumulative measurements during a visit. An important value for NEAT accuracy is what is obtained for an $R=6$ magnitude target: $0.8 \mu \mathrm{as} / \mathrm{h}$. The ratios between that (required) accuracy and the expected motions of the references indicates clearly that the latter cannot be considered as fixed. Their positions are members of the set of parameters that have to be solved for. Because the reference stars are much more distant $(\approx 1 \mathrm{kpc})$ than the target star $(\approx 10 \mathrm{pc})$, we are 100 times less sensitive to their planetary perturbations. Only Saturn-Jupiter mass objects matter, and statistically, they are only present around $\approx 10 \%$ of stars. These massive planets can be searched for by fitting first the reference star system $\left(\approx 100 N_{\text {ref }}\right.$ measurements for $5 N_{\text {ref }}$ parameters when there are no giant planets around the reference stars), possibly eliminate those with giant planets, and studying the target star with respect to that new reference frame. Moreover, the largest disturbers will be detected from ground based radial velocity measurements, and the early release of Gaia data around 2016 will greatly improve the position accuracy of the reference stars. For smaller planets at or below the threshold of detection, their impact on the target astrometry will be only at a level $\ll 1 \mathrm{M}_{\oplus}$ around it. Similarly the activity of these $\mathrm{K}$ giants has been investigated and neither the stellar pulsations nor the stellar spots will disturb the signal at the expected accuracy. 
Planetary system extraction from astrometric data. We recently carried out a major numerical simulation to test how well a space astrometry mission could detect planets in multi-planet systems (Traub et al. 2010). The simulation engaged 5 teams of theorists who generated model systems, and 5 teams of double-blind "observers" who analyzed the simulated data with noise included. The parameters of the study were the same as for NEAT, viz., astrometric singlemeasurement uncertainty $(0.80 \mu$ as noise, $0.05 \mu$ as floor, 5-year mission, plus RV observations with $1 \mathrm{~m} / \mathrm{s}$ accuracy for 15 years). We found that terrestrial-mass, habitable-zone planets $(\approx$ Earths) were detected with about the same efficiency whether they were alone in the system or if there were several other giant-mass, long-period planets $(\approx$ Jupiters) present. The reason for this result is that signals with unique frequencies are well separated from each other, with little cross-talk. The number of planets per system ranged from 1 to 11 , with a median of 3 . The SNR value of 5.8 value was predicted by Scargle (1982) for a false alarm probability (FAP) of less than 1\%, and verified in our simulations. The completeness and reliability to detect planets was better than $90 \%$ for all planets, where the comparison is with those planets that should have been detected according to a Cramer-Rao estimate (Gould et al., 2010) of the mission noise. The Cramer-Rao estimates of uncertainty in the parameters of mass, semi-major axis, inclination, and eccentricity were consistent with the observed estimates of each: $3 \%$ for planet mass, $\approx 4^{\circ}$ for inclination and 0.02 for eccentricity.

Radial velocity screening. To solve unambiguously for giant planets with periods longer than $5 \mathrm{yrs}$, it is necessary to have a ground RV survey for $15 \mathrm{yrs}$ of the 200 selected target star, at the presently available accuracy of $1 \mathrm{~m} / \mathrm{s}$. More than $80 \%$ of our targets are already being observed by RV, but the observations of the rest of them should start soon, well before the whole NEAT data is available. The capability of ground based RV surveys, despite their impressive near-term potential to obtain accuracies better than $1 \mathrm{~m} / \mathrm{s}$, is not sufficient to detect terrestrial planets in the $\mathrm{HZ}$ of $\mathrm{F}$, $\mathrm{G}$ and $\mathrm{K}$ stars. Formally, an accuracy of $0.05 \mathrm{~m} / \mathrm{s}$ is required to see an edge-on Earth mass planet at $1 \mathrm{AU}$ from a solar-mass star with $\mathrm{SNR}=5$ (semi-amplitude $=0.13 \mathrm{~m} / \mathrm{s}$ ), which might be achievable instrumentally, but is stopped in most cases by the impact of stellar activity on RV accuracy. It is necessary to find particularly "quiet" stars, but they are a minority (few percents) and cannot provide a full sample. Furthermore, the ambiguity in physical mass associated with the signal coming only from the radial component of the stellar reflex motion ( $\sin i$ ambiguity) requires additional in- formation to determine the physical mass and relative inclination in complex planetary systems. In some, but not all cases, limits are possible, and one can argue statistically that $90 \%$ of systems should be oriented such that the physical planet mass is within a factor of two of the mass found in RV. However, for finding a small number of potential future targets for direct detection and spectroscopy, an absolute determination that the mass is Earth-like is required as well as an exhaustive inventory of the planets around stars in our neighborhood.

Flexibility of objectives to upgrades / downgrades of the mission. One of the strengths of NEAT is its flexibility, the possibility to adjust the size of the instrument with impacts on the science that are not prohibitive. The size of the NEAT mission could be reduced (or increased) with a direct impact on the accessible number of targets but not in an abrupt way. For instance, for same amount of integration time and number of maneuvers, the options listed in Table 3 are possible, with impacts on the number of stars that can be investigated down to 0.5 and 1 Earth mass, and on the mass of the instrument, required fuel for maneuvers, and therefore cost. The time necessary to achieve a given precision depends on the mass limit that we want to reach: going from $0.5 \mathrm{M}_{\oplus}$ to $1 \mathrm{M}_{\oplus}$ requires twice less precision and therefore 4 times less observing time allowing a smaller telescope. There is room for adjustment keeping in mind that one wants to survey the neighborhood with the smallest mass limit possible and a typical number of targets of $\approx 200$.

\subsection{Technical issues}

Optical aberrations. NEAT uses a very simple telescope optical design. A 1-m diameter clear aperture offaxis parabola, with an off-axis distance of $1 \mathrm{~m}$ and a $40 \mathrm{~m}$ focal length. The focal plane is at the prime focus. The telescope is diffraction limited at the center of the field, where the target stars will be observed, but coma produces some field dependent aberrations. At the mean position of the reference stars, $0.2^{\circ}$ away from the center of the field, the coma produces a steady $23 \%$ increase of the point spread function (PSF) width and an $8 \mu \mathrm{m}$ centroid offset. The impact remains low since we are looking at differential effects.

Centroid measurements. They consist of two steps: the determination of the stellar centroid on each CCD during $57 \mathrm{~s}$ and then the calibration of the relative position of the CCDs during $3 \mathrm{~s}$ thanks to the metrology. The metrology determines also the response map of the detectors. As in the normal approach to precision astrometry with CCDs, we perform a least-square fit of 
Table 3 Science impact of NEAT scaling. The nominal mission is highlighted in yellow.

\begin{tabular}{|c|c|c|c|c|c|c|c|c|c|}
\hline \multirow[t]{2}{*}{$\begin{array}{c}\text { Mission } \\
\text { name }\end{array}$} & \multirow{2}{*}{$\begin{array}{c}\begin{array}{c}\text { Mirror } \\
\text { diameter }\end{array} \\
(\mathrm{m})\end{array}$} & \multirow{2}{*}{$\begin{array}{r}\begin{array}{r}\text { Focal } \\
\text { length }\end{array} \\
(\mathrm{m}) \\
\end{array}$} & \multirow{2}{*}{$\begin{array}{c}\begin{array}{c}\text { Field of view } \\
\text { diameter }\end{array} \\
(\operatorname{deg})\end{array}$} & \multirow{2}{*}{$\begin{array}{c}\begin{array}{c}\text { Focal Plane } \\
\text { size }\end{array} \\
(\mathrm{cm}) \\
\end{array}$} & \multirow{2}{*}{$\begin{array}{c}\begin{array}{c}\text { Ref. star mean } \\
\text { magnitude }\end{array} \\
\text { (R mag) }\end{array}$} & \multirow{2}{*}{$\begin{array}{l}\text { DMA } \\
\text { in } \mathbf{1 h} \\
\text { ( } \mu \mathrm{as}) \\
\end{array}$} & \multicolumn{3}{|c|}{$\begin{array}{l}\text { \# targets for a given } \\
\text { mass limit }\end{array}$} \\
\hline & & & & & & & $0.5 M \oplus$ & $1 M \oplus$ & $5 M_{\oplus}$ \\
\hline NEAT plus & 1.2 & 50 & 0.45 & 40 & 11.5 & 0.7 & 7 & 100 & 200 \\
\hline NEAT & 1.0 & 40 & 0.56 & 40 & 11 & 0.8 & 5 & 70 & 200 \\
\hline NEAT light & 0.8 & 30 & 0.71 & 35 & 10.5 & 1.0 & 4 & 50 & 200 \\
\hline EXAM & 0.6 & 20 & 0.85 & 30 & 10.1 & 1.4 & 2 & 35 & 200 \\
\hline
\end{tabular}

DMA $=$ Differential astrometric Measurement Accuracy (rms)

a template PSF to the pixelated data. PSF knowledge error leads to systematic errors in the conventional centroid estimation. We have developed an accurate centroid estimation algorithm by reconstructing the PSF from well sampled (above Nyquist frequency) pixelated images. In the limit of an ideal focal plane array whose pixels have identical response function (no inter-pixel variation), this method can estimate centroid displacement between two 32x32 images to sub-micropixel accuracy. Inter-pixel response variations exist in real CCDs, which we calibrate by measuring the pixel response of each pixel in Fourier space ${ }^{11}$. Capturing inter-pixel variations of pixel response to the third order terms in the power series expansion, we have shown with simulated data that the centroid displacement estimation is accurate to a few micro-pixels.

Stability of the primary mirror. The primary optic will be made of zerodur/ULE with a temperature coefficient better than $10^{-8} / \mathrm{K}$ with an optics thickness $\approx 10 \mathrm{~cm}$ and the effective temperature and temperature gradients are kept stable to $\approx 0.1 \mathrm{~K}$ over the mirror, the optic is then stable to $\approx 0.1 \mathrm{~nm}(\lambda / 6000)$ during the 5 yr mission. We have simulated two images, one at the center of the field that is a perfect Airy function and one at the edge of the field that has a $\lambda / 20$ coma. We added also wavefront errors with a conservative rms value of $\lambda / 1000$. With the new wavefronts, we calculated the change in the differential astrometry bias caused by both pixelation and changing wavefronts. While the wavefront deviations to optimal shape caused a centroid shift of $\approx 6-10 \mu$ as $\left(10^{-4}\right.$ pixels $)$, differential errors remained less than $\approx 0.3 \mu$ as $\left(3 \times 10^{-6}\right.$ pixels).

CCD damage in L2 environment. CCDs, like most semiconductors, suffer damage in radiation environments such as encountered by space missions. One particular performance parameter, Charge Transfer Efficiency (CTE), degrades with known consequences on

11 They are determined by calculating the first 6 coefficients of the Taylor series expansion in powers of wave numbers of the detector response map Fourier components. the efficiency of science missions like Gaid ${ }^{12}$ The reduced CTE is caused generally by prompt particle events (PPE), including solar protons and cosmic rays, colliding with the CCD silicon lattice and causing damages to the silicon lattice. This leads to the formation of so-called traps which can capture photo-electrons and release them again after some time. This results in signal loss and distortion of the PSF shape. The latter leads to systematic errors in the image location due to a mismatch between the ideal PSF shape and the actual image shape. For Gaia, this effect of radiation damage is a major contributor to the error budget and extensive research and laboratory tests have been done in order to understand better the radiation damage effects and to develop approaches in both hardware and data processing to mitigate the negative impact. However, there are a number of important differences between NEAT and Gaia which justify the assumption that radiation damage effects will play a much smaller role: i) NEAT looks for extended periods at very bright stars compared to Gaia in which the stars continuously move on the CCD. Also, unlike Gaia, NEAT will not be operated in time-delayed integration mode. In addition the CCDs are regularly illuminated by the laser light from the metrology system. This means that in general the signal level in the CCD pixels is high which will keep the traps with long $(\geq 60 \mathrm{~s})$ release time constants filled and effectively inactive. ii) NEAT also does not suffer from the varying CCD illumination history that a scanning mission like Gaia necessarily encounters. This illumination history is in fact one of the major complicating factors for Gaia. Finally, iii) NEAT uses much smaller CCDs than Gaia and in addition has four readout nodes, thus reducing the number of charge transfer steps and mitigating the effects of radiation damage. The one concern for the NEAT case is the presence of traps with release time constants that are of the order of several times the charge transfer period between pixels. In the case of NEAT the transfer period averages

12 The Gaia community (http://www.rssd.esa.int/gaia) speaks of the complementary quantity, charge transfer inefficiency (CTI), in order to emphasize its detrimental effects. 
tens of $\mu$ s and from laboratory tests with E2V CCDs, carried out in the context of the Gaia project, traps with time constants of $10-100 \mu$ s are known to exist. If these traps dominate at the operating temperature of the NEAT CCDs they could lead to subtle PSF image shape distortions and thus image location biases. From the Gaia experience, it is known that such image shape distortions can be handled in the post-processing by a careful modeling of the effects of radiation damage on the PSF image. A similar strategy, building on the Gaia heritage, can be employed for NEAT.

CCD/metrology tests in the lab. In the absence of optical errors, the major error sources are associated with the focal plane: (1) motions of the CCD pixels, which have to be monitored to $3 \times 10^{-6}$ pixels every $60 \mathrm{~s}$, i.e. $0.03 \mathrm{~nm}$; (2) measurements of the centroid of the star images with $5 \times 10^{-6}$ pixel accuracy. We have set up technology testbeds to demonstrate that we can achieve these objectives. The technology objective for (1) has almost been reached and the technology demonstration for (2) is underway and should be completed soon. Latest results with no metrology nor QE 6-parameter calibration have been obtained from the CCD / metrology test bench (Fig. 13). Allen deviation of the centroid location for one artificial star "A" projected on the CCD and Allen deviation of the differential centroid location for two artificial stars A and B projected on the CCD are plotted in Fig. 13 (left). One can see that star A moves on the CCD by a few hundred micro-pixels at time scale greater than 1 second, but that the differential position of the two stars is better $4 \times 10^{-5}$ pixel at 100 s integration time. On Fig. 13 (right), the Allen deviation of the differential centroid location for two artificial stars projected on the CCD is plotted, concatenating data from 38 runs, a minimum of $\approx 20 \mu$-pixels at about $10 \mathrm{~min}$ before differential drift dominated. This data shows that we are only a factor 10 from the final goal and that differential metrology at intervals of minutes is required to reach it.

\section{Perspectives}

In the Cosmic Vision plan for 2015-2025, the community has identified in Theme 1 the question: "What are the conditions for planet formation?", and the recommendation in Sect. 1.2: "Search for planets around stars other than the Sun..." ultra high precise astrometry as a key technique to explore our solar-like neighbors.

"On a longer timescale, a complete census of all Earth-sized planets within 100 pc of the Sun would be highly desirable. Building on Gaia's expected contribution on larger planets, this could be achieved with a high-precision terrestrial planet astrometric surveyor."

We have designed NEAT to be this astrometric surveyor. In Europe, as discussed in detail in the conclusions of the conference Pathways to Habitable Planets (Coudé Du Foresto et al. 2010) and in the Blue Dot Team report, the exoplanet community recognizes the importance of astrometric searches for terrestrial planets and has prioritized this search as a key question in the mid-term, i.e. in the time frame 2015-2022. The ExoPlanet Task Force (ExoPTF) in the US made a similar statement. Finally the ESA dedicated ExoPlanetary Roadmap Advisory Team (EPRAT) prioritizes Astrometric Searches for Terrestrial Planets in the mid term, i.e. in the time frame 2015-2022. Although the Decadal Survey of Astronomy and Astrophysics for 2010-2020 ranked down the SIM-Lite proposal, but placed as number one priority a program "to lay the technical and scientific foundation for a future mission to study nearby Earth-like planets".

Because of these recommandations by the community, we believe that there is a place for a mission like NEAT in future space programs, that is to say, a mission that is capable of detecting and characterizing planetary systems orbiting bright stars in the solar neighborhood that have a planetary architecture like that of our Solar System or an alternative planetary system partly composed of Earth-mass planets. These stars visible with the naked eye or simple binoculars, if found to host Earth-mass planets, will change humanity's view of the night sky.

Acknowledgements This work has benefited support from the Centre National des Études Spatiales (CNES), the Jet Propulsion Laboratory (JPL), Thales Alenia Space (TAS) and Swedish Space Corporation (SSC).

\section{References}

Coudé du Foresto V., Gelino D.M., Ribas I., Pathways Towards Habitable Planets, ASPC Series 430 (2010)

Erlig H, Qiu Y., Poberezhskiy I., Meras P., Wu J., Reliable optical pump architecture for highly coherent lasers used in space metrology applications, SPIE 7734, E71 (2010)

Gould A., Dong S., Gaudi B.S., Udalski A., Bond I.A., Greenhill J., Street R.A., Dominik M., Sumi T., Szymański M.K., Han C., et al. Frequency of Solar-like Systems and of Ice and Gas Giants Beyond the Snow Line from High-magnification Microlensing Events in 2005-2008, ApJ 720, 1073 (2010)

Guyon O., Shaklan S., Levine M., Cahoy K., Tenerelli D., Belikov R., Kern B., The pupil mapping exo- 

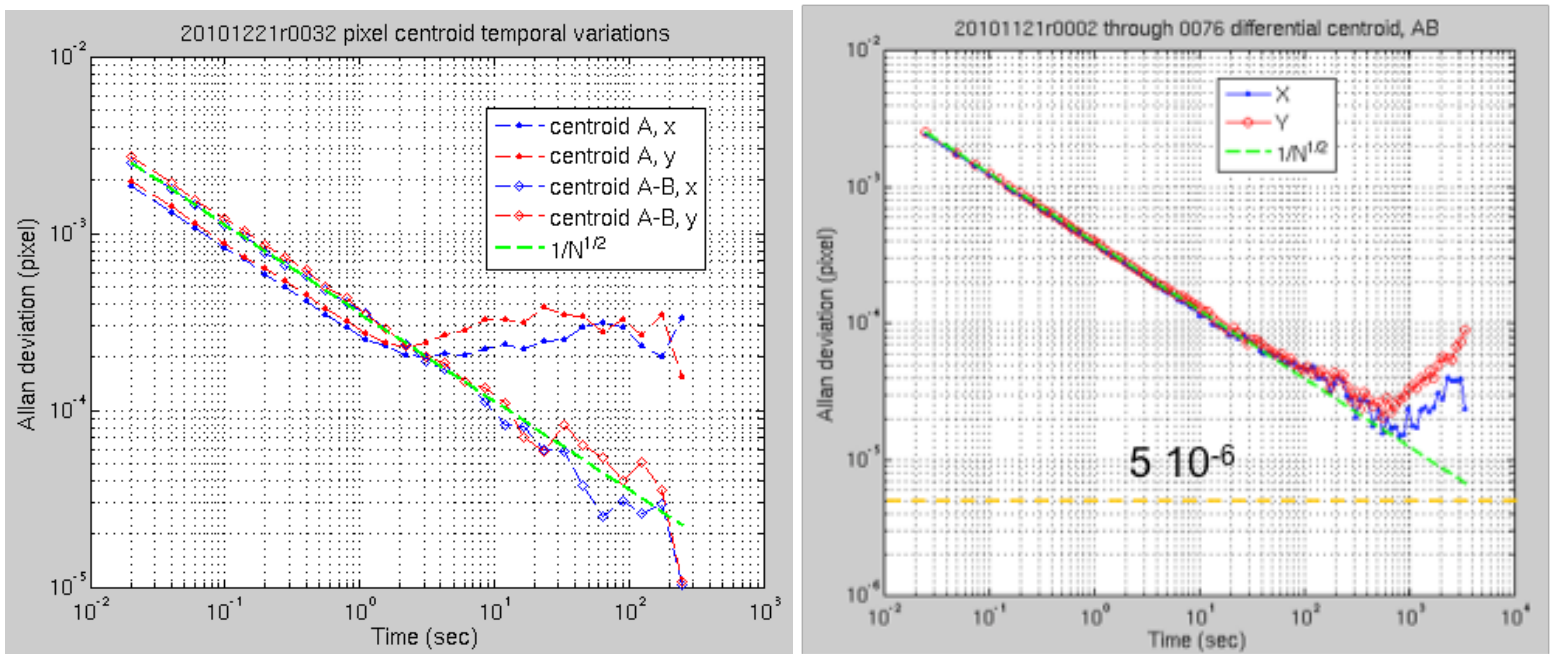

Fig. 13 Latest results obtained from the CCD/metrology test bench. No metrology nor quantum efficiency 6-parameter calibration have been performed yet. Left: Allen deviation of the centroid location for one artificial star "A" projected on the $\mathrm{CCD}$ and Allen deviation of the differential centroid location for two artificial stars A and B projected on the CCD. One can see that star A moves on the CCD by a few hundred micro-pixels at time scale greater than 1 second, but that the differential position of the two stars is better $410^{-5}$ pixel at $100 \mathrm{~s}$ integration time. Right: Allen deviation of the differential centroid location for two artificial stars projected on the CCD. Concatenating data from 38 runs, a minimum of $\approx 20 \mu$ pixels at about 10 min before differential drift dominated. This data shows that differential metrology at intervals of minutes is needed.

planet coronagraphic observer (PECO), SPIE 7731, 68 (2010)

Kaltenegger L., Eiroa C., Ribas I., Paresce F., Leitzinger M., Odert P., Hanslmeier A., et al. Stellar Aspects of Habitability - Characterizing Target Stars for Terrestrial Planet-Finding Missions, Astrobiology 10,103 (2010)

Lagrange A., Meunier N., Desort M., Malbet F. Using the Sun to estimate Earth-like planets detection capabilities . III. Impact of spots and plages on astrometric detection, A\&A 528, L9 (2011)

Makarov V.V., Beichman C.A., Catanzarite J.H., Fischer D.A., Lebreton J., Malbet F., Shao M. Starspot Jitter in Photometry, Astrometry, and Radial Velocity Measurements, ApJL 707, L73 (2009)

Makarov V.V., Parker D., Ulrich R.K. Astrometric Jitter of the Sun as a Star, ApJ 717, 1202 (2010)

Meunier N., Lagrange A., Desort M. Reconstructing the solar integrated radial velocity using $\mathrm{MDI} / \mathrm{SOHO}$, A\&A 519, A66 (2010)

Scargle J.D. Studies in astronomical time series analysis. II - Statistical aspects of spectral analysis of unevenly spaced data. ApJ 263, 835 (1982)

Traub W.A., Beichman C., Boden A.F., Boss A.P., Casertano S., Catanzarite J., Fischer D., et al. Detectability of Earth-like Planets in Multi-Planet Systems: Preliminary Report. EAS Pub Series 42, 191 (2010)

van Leeuwen F. Validation of the new Hipparcos reduction. A\&A 474, 653 (2007)

\section{Author affiliations}

F. Malbet - Ph. Feautrier - A.-M. Lagrange - A. Chelli - G. Duvert - T. Forveille - N. Meunier : UJFGrenoble 1 / CNRS-INSU, Institut de Planétologie et d'Astrophysique de Grenoble (IPAG), UMR 5274, BP 53, F-38041 Grenoble cedex 9, France. E-mail: Fabien.Malbet@obs.ujf-grenoble.fr

A. Léger : Université Paris Sud / CNRS-INSU, Institut d'Astrophysique Spatiale (IAS) UMR 8617, Bât. 120-121, F-91405 Orsay cedex, France

M. Shao - R. Goullioud - W. Traub : Jet Propulsion Laboratory (JPL), California Institute of Technology, 4800 Oak Grove Drive, Pasadena CA 91109, USA

P.-O. Lagage - C. Cara - G. Durand : Laboratoire AIM, CEA-IRFU / CNRS-INSU / Université Paris Diderot, CEA Saclay, Bât 709, F-91191 Gifsur-Yvette Cedex, France

A.G.A. Brown - H.J.A. Röttgering : Leiden Observatory, Leiden University, P.O. Box 9513, NL-2300 RA Leiden, The Netherlands

C. Eiroa - J. Maldonado - E. Villaver : Universidad Autónoma de Madrid (UAM), Dpto. Física Teórica, Módulo 15, Facultad de Ciencias, Campus de Cantoblanco, E-28049 Madrid, Spain 
B. Jakobsson : Swedish Space Corporation (SSC), P.O. Box 4207, SE-17104 Solna, Sweden

E. Hinglais : Centre National d'Etudes Spatiales (CNES), Centre spatial de Toulouse, 18 avenue Edouard Belin, F-31401 Toulouse cedex 9, France

L. Kaltenegger - C. Mordasini : Max-Planck-Institut für Astronomie, Königstuhl 17, D-69117 Heidelberg, Germany

L. Labadie : I. Physikalisches Institut der Universität zu Köln, Zülpicher Str. 77, D-50937 Köln, Germany

J. Laskar - N. Rambaux : UPMC-Paris 6 / Observatoire de Paris / CNRS-INSU, Institut de mécanique céleste et de calcul des éphémérides (IMCCE) UMR 8028, 77 avenue Denfert-Rochereau, F-75014 Paris, France

R. Liseau : Chalmers University of Technology, SE41296 Gothenburg, Sweden

J. Lunine : Dipartimento di Fisica, University of Rome Tor Vergata, Via della Ricerca Scientifica 1, Roma I-00133, Italy

M. Mercier : Thales Alenia Space, 100 boulevard du Midi, F-06150 Cannes, France

D. Queloz - J. Sahlmann - D. Ségransan : Observatoire Astronomique de l'Université de Geneve, 51 Chemin des Maillettes, CH-1290 Sauverny, Switzerland

A. Quirrenbach : Universität Heidelberg, Landessternwarte, Königstuhl 12, D-69117 Heidelberg, Germany

A. Sozzetti - A.H. Andrei : INAF - Osservatorio Astronomico di Torino, Strada Osservatorio 20, I10025 Pino Torinese, Italy

O. Absil - J. Surdej : Université de Liège, Département d'Astrophysique, Géophysique et Océanographie, 17 Allée du Six Août, B-4000 Sart Tilman, Belgium

Y. Alibert : Physikalisches Institut, University of Bern, Sidlerstrasse 5, 3012, Bern, Switzerland

Y. Alibert : Université de Besançon / Observatoire de Besançon / CNRS-INSU UMR 6213, Institut UTINAM, 41 bis Avenue de l'Observatoire, BP 1615, F-25010 Besançon cedex, France
A.H. Andrei : Observatorio Nacional - Ministério da Ciência e Tecnologia, Rua General Cristino 77, Sāo Cristóvāo 20921-400, Rio de Janeiro, Brazil

F. Arenou : Université Paris 7 Diderot / Observatoire de Paris / CNRS-INSU, UMR 8111, "Galaxie Etoile Physique Instrumentation" (GEPI), 5 Place Jules Janssen, F-92190 Meudon cedex, France

C. Beichman : California Institute of Technology, NASA Exoplanet Science Institute / IPAC, 770 South Wilson Ave, Pasadena CA 91125, USA

C.S. Cockell : The Open University, Dept. of Physics \& Astronomy, Planetary and Space Sciences Research Institute, Milton Keynes MK7 6AA, UK

P.J.V. Garcia : Universidade do Porto, Faculdade de Engenharia, Departamento de Engenharia Fsica, Laboratrio SIM, Rua Dr. Roberto Frias, P-4200-465 Porto, Portugal

D. Hobbs : Lund Observatory, Lund University, Box 43, SE-22100 Lund, Sweden

A. Krone-Martins : Instituto de Astronomia, Geofísica e Ciências Atmosféricas, Universidade de São Paulo, Rua do Matão, 1226, Cidade Universitária, 05508900 São Paulo-SP, Brazil

A. Krone-Martins - S. Raymond - F. Selsis : Université de Bordeaux 1 / Observatoire Aquitain des Sciences de l'Univers / CNRS-INSU, UMR 5804, Laboratoire d'Astrophysique de Bordeaux, BP 89, F-33271 Floirac Cedex, France

H. Lammer : Space Research Institute, Austrian Academy of Sciences, Schmiedlstr. 6, A-8042 Graz, Austria

S. Minardi : Institute of Applied Physics, Friedrich Schiller, University Jena, Max-Wien-Platz 1, D-07743 Jena, Germany

A. Moitinho de Almeida - A. Krone-Martins : Systems, Instrumentation and Modeling (SIM) - Faculdade de Ciências da Universidade de Lisboa, Ed. C8, Campo Grande, 1749-016 Lisboa, Portugal

P.A. Schuller : Université Paris 7 Diderot / Université Pierre et Marie Curie / Observatoire de Paris / CNRS-INSU, UMR 8109, Laboratoire d'études spatiales et d'instrumentation en astrophysique (LESIA), 5 Place Jules Janssen, F-92190 Meudon cedex, France 
G.J. White : The Open University, Dept. of Physics \& Astronomy, Venables Building, Walton Hall, Milton Keynes MK7 6AA, UK

G.J. White : Rutherford Laboratory, Space Science \& Technology Department, CCLRC Rutherford Appleton Laboratory, Chilton, Didcot, Oxfordshire OX11 0QX, UK

H. Zinnecker : Deutsches SOFIA Institut, Institut für Raumfahrtsysteme, Universität Stuttgart, Pfaffenwaldring 31, D-70569 Stuttgart, Germany

H. Zinnecker : SOFIA Science Center, NASA-Ames, MS 211-3,Moffett Field, CA 94035, USA 